\title{
A BETA FAMILY IN THE HOMOTOPY OF SPHERES
}

\author{
KATSUMI SHIMOMURA
}

(Communicated by Brooke Shipley)

\begin{abstract}
Let $p$ be a prime number greater than three. In the $p$-component of stable homotopy groups of spheres, Oka constructed a beta family from a $v_{2}$-periodic map on a four cell complex. In this paper, we construct another beta family in the groups at a prime $p$ greater than five from a $v_{2}$-periodic map on an eight cell complex.
\end{abstract}

\section{INTRODUCTION}

We fix a prime number $p$ greater than three and work in the stable homotopy category $\mathcal{S}_{(p)}$ of spectra localized at the prime $p$. Let $S$ and $B P$ in $\mathcal{S}_{(p)}$ denote the sphere and the Brown-Peterson spectra. It is important to understand the homotopy groups $\pi_{*}(S)$, whose structure is little known. On the other hand, we know the structures of $\pi_{*}(B P)=B P_{*}$ and $B P_{*}(B P)$ :

$$
B P_{*}=\mathbb{Z}_{(p)}\left[v_{1}, v_{2}, \ldots\right] \text { and } B P_{*}(B P)=B P_{*}\left[t_{1}, t_{2}, \ldots\right]
$$

and $B P_{*}(B P)$ is a Hopf algebroid over $B P_{*}$. Here, the generators have degrees $\left|v_{k}\right|=\left|t_{k}\right|=2\left(p^{k}-1\right)$. Furthermore, we have the Adams-Novikov spectral sequence converging to the homotopy groups $\pi_{*}(X)$ of a spectrum $X$ with $E_{2}$-term

$$
E_{2}^{s, t}(X)=\operatorname{Ext}_{B P_{*}(B P)}^{s, t}\left(B P_{*}, B P_{*}(X)\right),
$$

and the spectral sequence for $X=S$ acts as a go-between between $B P$ and $S$. Here we consider the homotopy groups $\pi_{*}(S)$ through the spectral sequence. In the $E_{2}$-term $E_{2}^{2, *}(S)$, Miller, Ravenel and Wilson 1 defined the beta elements $\beta_{s / t, r}$ for suitable triples $(s, t, r)$ of positive integers. In this paper, we merge the methods of Oka and the authors to find permanent cycles among the beta elements and obtain a family of beta elements in the homotopy groups of the sphere spectrum.

Theorem. Let $p$ be a prime number greater than five. We have following beta elements in the homotopy groups $\pi_{*}(S)$ detected by the beta elements in the $E_{2}$ term:

a) $\beta_{s p^{n} / t} \in \pi_{\left(s p^{n}(p+1)-t\right) q-2}(S)$ for $s \geq 1, n \geq 2,1 \leq t \leq 2^{n-2} p^{2}-2$ and

b) $\beta_{s p^{n} / u p, 2} \in \pi_{\left(s p^{n}(p+1)-u p\right) q-2}(S)$ for $s \geq 1, n \geq 3,1 \leq u \leq 2^{n-3} p-1$.

The orders of elements of a) and $\mathrm{b}$ ) are no less than $p$ and $p^{2}$, respectively.

Received by the editors March 20, 2012 and, in revised form, August 13, 2012 and August 30, 2012.

2010 Mathematics Subject Classification. Primary 55Q45; Secondary 55Q51. 
From now on, we state the definitions, known results and theorems obtained in this paper. Consider the spectra and the maps defined by the cofiber sequences:

$$
\begin{gathered}
S \stackrel{p^{r}}{\longrightarrow} S \stackrel{i_{r}}{\longrightarrow} M(r) \stackrel{j_{r}}{\longrightarrow} \Sigma S \quad \text { and } \\
\Sigma^{u p^{r-1} q} M(r) \stackrel{A_{r-1}^{u}}{\longrightarrow} M(r) \stackrel{i_{r, u p^{r-1}}}{\longrightarrow} M\left(r, u p^{r-1}\right) \stackrel{j_{r, u p^{r-1}}}{\longrightarrow} \Sigma^{u p^{r-1} q} M(r),
\end{gathered}
$$

where $A_{r}$ denotes an element such that $B P_{*}\left(A_{r}\right)=v_{1}^{p^{r}}$ for $r \geq 0$ (cf. [6. Th. 6.2]; see also (2.6)), and $A_{0}$ is known as the Adams map and denoted by $\alpha$. Hereafter, $q=2 p-2$. We note that $B P_{*}(M(r))=B P_{*} /\left(p^{r}\right)$ and $B P_{*}\left(M\left(r, u p^{r-1}\right)\right)=$ $B P_{*} /\left(p^{r}, v_{1}^{u p^{r-1}}\right)$ as $B P_{*}(B P)$-comodules. The cofiber sequences in (1.1) induce the connecting homomorphisms $\partial_{r}: E_{2}^{s, t}(M(r)) \rightarrow E_{2}^{s+1, t}(S)$ and $\partial_{r, u p^{r-1}}$ : $E_{2}^{s, t}\left(M\left(r, u p^{r-1}\right)\right) \rightarrow E_{2}^{s+1, t-u p^{r-1} q}(M(r))$ on the $E_{2}$-terms. In [11, we modified the definition of the beta elements as follows: let $(s, t, r)$ be a triple of positive integers, and suppose that $v_{1}^{c} v_{2}^{s} \in E_{2}^{0,(s(p+1)+c) q}(M(r, t+c))$ with $p^{r-1} \mid(t+c)$ for an integer $c$. Then, the beta element for a triple $(s, t, r)$ is defined by

$$
\beta_{s / t, r}=\partial_{r} \partial_{r, t+c}\left(v_{1}^{c} v_{2}^{s}\right) \in E_{2}^{2,(s(p+1)-t) q}(S) .
$$

We abbreviate $\beta_{s / t, 1}$ and $\beta_{s / 1}$ to $\beta_{s / t}$ and $\beta_{s}$, respectively, as usual. In the case where $c=0$, the definition is the ordinary one. Besides, these elements generate the $E_{2}$-term by [1, Th. 2.6]. It is an interesting problem asking which of them survives in the spectral sequence. So far, the following elements are known to be permanent cycles:

a) $\beta_{s}$ for $s \geq 1$ in 12 ,

b) $\beta_{s p / t}$ for $s \geq 1$ and $t \leq p$, and $t<p$ if $s=1$ in [2, 3],

c) $\beta_{s p^{2} / t}$ for $s \geq 1$ and $t \leq 2 p$, and $t \leq 2 p-2$ if $s=1$ in [2], 4],

d) $\beta_{s p^{2} / t}$ for $s \geq 1$ and $t \leq p^{2}-2$ in [11,

e) $\beta_{s p^{n} / t}$ for $s \geq 1, n \geq 3,1 \leq t \leq 2^{n-2} p$, and $t \leq 2^{n-2}(p-1)$ if $s=1$ in [6, 7,

f) $\beta_{s p^{2} / p, 2}$ for $s \geq 2$ in [4, and

g) $\beta_{s p^{n} / u p, 2}$ for $s \geq 1, n \geq 3,1 \leq u \leq 2^{n-2}$, and $u p \leq 2^{n-2}(p-1)$ if $s=1$ in [6], 7].

We note that we have $\beta_{s p^{n} / t}$ for $t \leq p^{n}+p^{n-1}-1$ and $t \leq p^{n}$ if $s=1$ in the $E_{2}$-term by [1, and Ravenel showed that $\beta_{p^{n}} / p^{n}$ cannot be a permanent cycle for $n \geq 1$ (cf. [9, 6.4.2. Th.]). Thus, the beta elements $\beta_{s p^{n} / t}$ for $2^{n-2} p<t \leq p^{n}+p^{n-1}-1$ and $t<p^{n}$ if $s=1$ are left undetermined.

In this paper we modify the definition further.

Definition 1.4. Let $b(s ; t, r)$ denote a set of elements $x$ of $E_{2}^{0,(s(p+1)+c) q}(M(r, t+c))$ such that $x \equiv v_{1}^{c} v_{2}^{s} \bmod \left(p, v_{1}^{c+1}\right)$ for a non-negative integer $c$. We define the beta coset by

$$
\widehat{\beta}_{s / t, r}=\partial_{r} \partial_{r, t+c}(b(s ; t, r)) \subset E_{2}^{2,\left(s(p+1)+c-u p^{r-1}\right) q}(S) .
$$

We also abbreviate $\widehat{\beta}_{s / t, 1}$ to $\widehat{\beta}_{s / t}$.

In [6, Th. II], Oka showed a possibility of many beta elements in the same dimension. This indicates that a difference of elements of $\widehat{\beta}_{s / t, r}$ may be another beta element. In this paper, we study a beta element $\beta_{s / t, r}$ with a larger $t$, not the $p$-rank of $\pi_{*}(S)$, and we introduce the notation. We further abuse a term. 
Definition 1.5. We say that the beta coset $\widehat{\beta}_{s / t, r}$ survives to the homotopy groups $\pi_{*}(S)$ if an element of $\widehat{\beta}_{s / t, r}$ is a permanent cycle. In this case, the $\beta$-element $\beta_{s / t, r}$ of $\pi_{*}(S)$ denotes one of survivors of $\widehat{\beta}_{s / t, r}$.

In this paper, we consider the beta cosets $\widehat{\beta}_{s / t, r}$ for $r=1,2$, and so we consider the following spectra and maps of (1.1):

$$
M=M(1), \quad \bar{M}=M(2), \quad K_{u}=M(1, u) \quad \text { and } \quad \bar{K}_{u}=M(2, u p) ; \quad \text { and }
$$

$$
k=k_{1}, \quad \bar{k}=k_{2}, \quad \alpha=A_{0}, \quad A=A_{1}, \quad k_{u}=k_{1, u} \quad \text { and } \quad \bar{k}_{u}=k_{2, u p}
$$

for $u>0$, where $k$ stands for $i$ and $j$. (We use the notation $M$ and $K_{u}$ following those of Oka [7.) Thus, from now on, $i_{u}$ and $j_{u}$ denote $i_{1, u}$ and $j_{1, u}$, not the maps $i_{u}$ and $j_{u}$ in (1.1).

The above definitions make Oka's method developed in [6] and [7] simple: Let $f_{s, u} \in \pi_{*}\left(K_{u}\right)$ be an element such that $\eta_{*}\left(f_{s, u}\right)=v_{2}^{s} \in B P_{*}\left(K_{u}\right)=B P_{*} /\left(p, v_{1}^{u}\right)$ for the unit map $\eta: S \rightarrow B P$ of the ring spectrum $B P$, and put

$$
\begin{aligned}
& \mathfrak{B}_{\text {Oka }}(s, u)=\mathfrak{B}_{\text {Oka }}^{0}(s, u) \cup \mathfrak{B}_{\text {Oka }}^{1}(s, u), \text { where } \\
& \mathfrak{B}_{\text {Oka }}^{0}(s, u)=\left\{\widehat{\beta}_{s k p^{n} / t}: k \geq 1, n \geq 0,1 \leq t \leq 2^{n} u\right\}, \\
& \mathfrak{B}_{\text {Oka }}^{1}(s, u)=\left\{\widehat{\beta}_{s k p^{n} / t p, 2}: k \geq 1, n \geq 1, t \geq 1, t p \leq 2^{n-1} u\right\} .
\end{aligned}
$$

Theorem 1.7 (Oka [6], [7]). If $f_{s, u} \in \pi_{*}\left(K_{u}\right)$ exists, then every element of $\mathfrak{B}_{\text {Oka }}(s, u)$ survives to $\pi_{*}(S)$.

Consider $\overline{\mathfrak{B}}_{\text {Oka }}((a, b), u)=\bigcup_{k, l \geq 0, k+l>0} \mathfrak{B}_{\text {Oka }}(a k+b l, u)$. Since Oka also showed the existence of $f_{s p, u} \in \pi_{*}\left(K_{u}\right)$ for $s=2,3$ and $u \leq p$ and for $s=1$ and $u<p$ in [2, Th. C] and [3, Th. CII], the theorem implies that every element of $\mathfrak{B}_{O k a}(p, p-$ 1) $\cup \overline{\mathfrak{B}}_{O k a}((2 p, 3 p), p)$ survives to $\pi_{*}(S)$, which is the theorem [6, Th. I], and yields elements in b), c), e), f) and g) in (1.3).

Let $W$ be the cofiber of the generator $\beta_{1} \in \pi_{p q-2}(S)$, and we have a cofiber sequence

$$
S^{p q-2} \stackrel{\beta_{1}}{\longrightarrow} S^{0} \stackrel{i_{W}}{\longrightarrow} W \stackrel{j_{W}}{\longrightarrow} S^{p q-1} .
$$

In [10, we introduce a method to obtain a beta family from $f_{s, u} \in \pi_{*}\left(W \wedge K_{u}\right)$ such that $\eta_{*}\left(f_{s, u}\right)=v_{2}^{s} \in B P_{*}\left(W \wedge K_{u}\right)$. In this paper, we merge these methods. For an element $f_{p^{i}, u} \in \pi_{*}\left(W \wedge K_{u}\right)$, consider a family

$$
\begin{aligned}
\mathfrak{B}\left(p^{i}, u\right) & =\mathfrak{B}^{0}\left(p^{i}, u\right) \cup \mathfrak{B}^{1}\left(p^{i}, u\right), \quad \text { where } \\
\mathfrak{B}^{0}\left(p^{i}, u\right) & =\left\{\widehat{\beta}_{s p^{i+n} / t}: s \geq 1, n \geq 0,1 \leq t \leq 2^{n} u-2\right\}, \\
\mathfrak{B}^{1}\left(p^{i}, u\right) & =\left\{\widehat{\beta}_{s p^{i+n} / t p, 2}: s \geq 1, n \geq 1, t \geq 1, t p \leq 2^{n-1} u-p\right\} .
\end{aligned}
$$

Theorem 1.9. If $f_{p^{i}, u} \in \pi_{*}\left(W \wedge K_{u}\right)$ exists, then every element of $\mathfrak{B}\left(p^{i}, u\right)$ survives to $\pi_{*}(S)$.

In [11, Th. 1.7], we showed the existence of $f_{p^{2}, p^{2}} \in \pi_{*}\left(W \wedge K_{p^{2}}\right)$ for $p>5$, though there does not exist $f_{p^{2}, p^{2}} \in \pi_{*}\left(K_{p^{2}}\right)$ shown by Ravenel.

Corollary 1.10. Let $p>5$. Then, $\mathfrak{B}\left(p^{2}, p^{2}\right)$ yields a beta family of $\pi_{*}(S)$.

This implies our main theorem stated above. This improves Oka's results (1.3)e) and $\mathrm{g}$ ) if the prime number $p$ is greater than five. 


\section{RECOLLECTION OF THE FINITE RING SPECTRUM}

In this section, we recall some results of Oka. We call a spectrum $E$ a ring spectrum if it admits a multiplication $\mu: E \wedge E \rightarrow E$ and a unit $\iota: S \rightarrow E$ such that $\mu(\iota \wedge 1)=1=\mu(1 \wedge \iota)$ and $\mu(\mu \wedge 1)=\mu(1 \wedge \mu)$. A ring spectrum $E$ is commutative if $\mu T=\mu$ for the switching map $T: E \wedge E \rightarrow E \wedge E$. The homotopy groups $E_{*}=\pi_{*}(E)$ of $E$ have a multiplication given by $a b=\mu(a \wedge b)$ for $a, b \in E_{*}$, which makes $E_{*}$ a ring. Oka [7] (cf. [8]) defined $\operatorname{Mod}(E)$ and $\operatorname{Der}(E)$ by

$$
\begin{aligned}
\operatorname{Mod}(E) & =\left\{f \in[E, E]_{*} \mid \mu(f \wedge 1)=f \mu\right\} \quad \text { and } \\
\operatorname{Der}(E) & =\left\{f \in[E, E]_{*} \mid \mu(f \wedge 1)+\mu(1 \wedge f)=f \mu\right\} .
\end{aligned}
$$

We call an element of $\operatorname{Der}(E)$ a derivation of $E$.

Theorem 2.1 (Oka [8, Lemma 1.3]). For the unit $\iota$, the induced homomorphism $\iota^{*}: \operatorname{Mod}(E) \rightarrow E_{*}$ is a ring isomorphism. Its inverse $\kappa: E_{*} \rightarrow \operatorname{Mod}(E)$ is given by $\kappa(f)=\mu(f \wedge 1)$.

Consider a spectrum $K_{u}$ in (1.6). Then, Oka showed that

Theorem 2.2 (Oka [7, Th. 2.5]). $K_{u}$ has a commutative and associative multiplication $m_{u}$.

Theorem 2.3 (Oka [7, Lemma 2.3]). $\operatorname{Mod}\left(K_{u}\right)$ is a commutative subring of $\left[K_{u}\right.$, $\left.K_{u}\right]_{*}$, and a commutator $[f, g]$ belongs to $\operatorname{Mod}\left(K_{u}\right)$ for $f \in \operatorname{Mod}\left(K_{u}\right)$ and $g \in$ $\operatorname{Der}\left(K_{u}\right)$. In particular,

$$
f^{p} g=g f^{p} \quad \text { for } f \in \operatorname{Mod}\left(K_{u}\right) \text { and } g \in \operatorname{Der}\left(K_{u}\right) .
$$

Let $\delta_{u}^{\prime}=i_{u} j_{u} \in\left[K_{u}, K_{u}\right]_{-u q-1}$. Then it fits into a cofiber sequence

$$
\Sigma^{u q} K_{u} \stackrel{\widetilde{i}_{u}}{\longrightarrow} K_{2 u} \stackrel{\widetilde{j}_{u}}{\longrightarrow} K_{u} \stackrel{\delta_{u}^{\prime}}{\longrightarrow} \Sigma^{u q+1} K_{u}
$$

by (1.1) with a $3 \times 3$ Lemma.

Theorem 2.5 (Oka [7, Th. 2.5]). $\quad \delta_{u}^{\prime} \in \operatorname{Der}\left(K_{u}\right)$.

It is well known that $\delta=i j \in \operatorname{Der}(M)$ and $\alpha \in \operatorname{Mod}(M)$, and so $\alpha^{p} \delta=\delta \alpha^{p} \in$ $[M, M]_{p q-1}$. It gives rise to not only the element $A=A_{1}$ in (1.1) but also $\delta_{u}$ in the commutative diagram

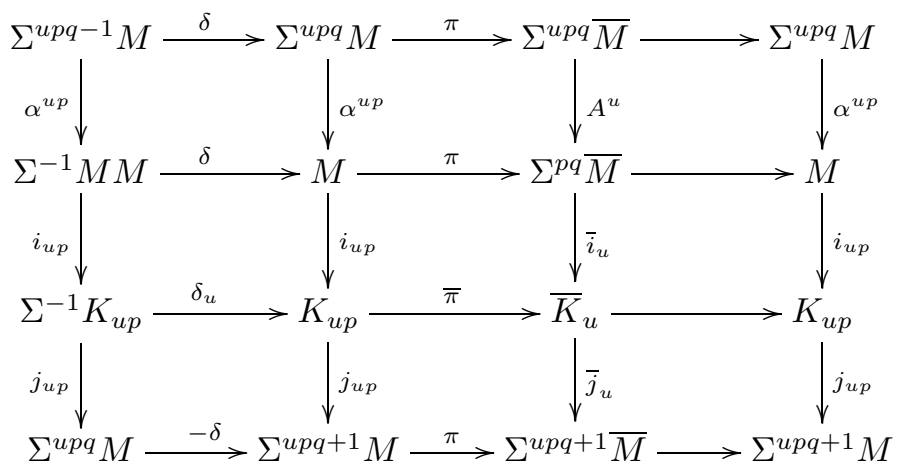

in which rows and columns are cofiber sequences. By [6, Lemma 4.5, Th. 4.2], we have the following: 
Theorem 2.7 (Oka [7, p. 425]). The map $\delta_{u}$ in the above diagram is a derivation of $K_{u p}$.

Proof. The matrices for the map $\delta_{u} \wedge 1$ and the switching map $T$ are given by

$$
\tau\left(\delta_{u} \wedge 1\right)=\left(\begin{array}{cccc}
0 & 1 & 0 & 0 \\
0 & 0 & \beta & 0 \\
0 & 0 & 0 & -1 \\
0 & 0 & 0 & 0
\end{array}\right) \quad \text { and } \quad \tau(T)=\left(\begin{array}{cccc}
1 & 0 & 0 & 0 \\
\delta & -1 & 0 & 0 \\
\delta^{\prime} & 0 & -1 & 0 \\
\delta \delta^{\prime} & \delta^{\prime} & -\delta & 1
\end{array}\right)
$$

by [6, Lemma 4.5] and [6, Th. 4.2], and so the first row of the matrix for $\left(\delta_{u} \wedge\right.$ $1)+\left(\delta_{u} \wedge 1\right) T$ is $\left(\begin{array}{llll}\delta & 0 & 0 & 0\end{array}\right)$. Since the multiplication $m_{u}$ is the projection to the first summand, we see that $m_{u}\left(\delta_{u} \wedge 1+1 \wedge \delta_{u}\right)=m_{u}\left(\left(\delta_{u} \wedge 1\right)+T\left(\delta_{u} \wedge 1\right) T\right)=$ $m_{u}\left(\left(\delta_{u} \wedge 1\right)+\left(\delta_{u} \wedge 1\right) T\right)=\delta_{u} m_{u}$, as desired.

The following lemma is a folklore:

Lemma 2.8. There exist self-maps $\widetilde{\alpha}: \Sigma^{q} K_{u} \rightarrow K_{u}$ and $\widetilde{A}: \Sigma^{p q} \bar{K}_{u} \rightarrow \bar{K}_{u}$ such that $B P_{*}(\widetilde{\alpha})=v_{1}$ and $B P_{*}(\widetilde{A})=v_{1}^{p}$.

Lemma 2.9. $A \bar{i} \beta_{1}=0 \in \pi_{2 p q-2}(\bar{M})$.

Proof. Consider the cobar complex $\left\{\left(C^{s}, d\right)\right\}_{s \geq 0}$ whose cohomology is the $E_{2}$-term $E_{2}^{*}(\bar{M})$ of the Adams-Novikov spectral sequence converging to $\pi_{*}(\bar{M})$. Then, $C^{s}=\Gamma /\left(p^{2}\right) \otimes_{A} \Gamma^{s-1}$, where $(A, \Gamma)=\left(B P_{*}, B P_{*} B P\right)$, and the differential $d$ of the complex is given by derivation with $d(v)=\eta_{R}(v)-\eta_{L}(v) \in C^{1}=\Gamma /\left(p^{2}\right)$ for $v \in C^{0}=A /\left(p^{2}\right)$ and $d(x)=1 \otimes x-\Delta(x)+x \otimes 1 \in C^{2}$ for $x \in C^{1}$. We also use the formulas on the structure maps of the Hopf algebroid given by the formulas of Quillen and Hazewinkel:

$$
\begin{aligned}
& \eta_{R}\left(v_{1}\right)=v_{1}+p t_{1}, \quad \eta_{R}\left(v_{2}\right) \equiv v_{2}+v_{1} t_{1}^{p}+p t_{2}-(p+1) v_{1}^{p} t_{1} \quad \bmod \left(p^{2}\right), \\
& \Delta\left(t_{1}\right)=1 \otimes t_{1}+t_{1} \otimes 1 \quad \text { and } \Delta\left(t_{2}\right)=1 \otimes t_{2}+t_{1} \otimes t_{1}^{p}+t_{1} \otimes 1+v_{1} b_{10} .
\end{aligned}
$$

Here, $b_{10}$ denotes the cocycle defined by $d\left(t_{1}^{p}\right)=p b_{10}$. Let $b_{0}$ denote the cohomology class of $b_{10}$. Then, by definition, $\beta_{1}=\partial_{1} \partial_{1,1}\left(v_{2}\right)=b_{0}$. Therefore, $A \bar{i} \beta_{1}$ is detected by $v_{1}^{p} b_{0} \in E_{2}^{2,2 p q}(\bar{M})$. We compute that $d(c)=v_{1}^{p} b_{10} \in C^{2}$ for $c=-v_{1}^{p-1} t_{2}+v_{1}^{p-3}\left(v_{1} t_{1}-p t_{1}^{2}\right) \eta_{R}\left(v_{2}\right)+v_{1}^{2 p-3}\left(\frac{p+1}{2} v_{1} t_{1}^{2}-\frac{p}{3} t_{1}^{3}\right)$. It follows that $v_{1}^{p} b_{0}=$ $0 \in E_{2}^{2,2 p q}(\bar{M})$. We further see that $E_{2}^{2+r q, 2 p q+r q}(\bar{M})=0$ for $r \geq 1$ by the vanishing line (cf. [1, Lemma 1.16, Remark 1.17]). Hence $A \bar{i} \beta_{1}=0 \in \pi_{2 p q-2}(\bar{M})$.

\section{Arguments on $W \wedge K_{u}$}

In this section, we fix an integer $u$, and $K$ denotes $K_{u}=M(1, u)$, which is a commutative ring spectrum with multiplication $m=m_{u}$ by Theorem 2.2. The spectrum $W$ in (1.8) admits a multiplication $m_{W}: W \wedge W \rightarrow W$ such that $m_{W}\left(i_{W} \wedge\right.$ $\left.1_{W}\right)=1_{W}=m_{W}\left(1_{W} \wedge i_{W}\right)$ by [5. Example 2.9].

Consider the spectrum $W M=W \wedge M$ and the multiplication $m_{W M}=\left(m_{W} \wedge\right.$ $\left.m_{M}\right)\left(1_{W} \wedge T \wedge 1_{M}\right): W M \wedge W M \rightarrow W M$. Then, we see that

$$
m_{W M}\left(i^{\prime} \wedge i^{\prime}\right)=i^{\prime} m_{W},
$$

for $i^{\prime}=1_{W} \wedge i$. We further see the following lemma by [6, Th. 4.13]:

Lemma 3.2. WM is a commutative and associative ring spectrum with multiplication $m_{W M}$. 
Proof. Since $p=0 \in[W M, W M]_{0}$ and $\beta_{1} \wedge 1=0 \in[M \wedge W M, M \wedge W M]_{p q-2}$, $W M$ is a split ring spectrum. (See [6, Def. 2.1] for the definition of a split ring spectrum.)

Put $W M K=W \wedge M \wedge K$, and consider a multiplication $m_{W M K}=\left(m_{W M} \wedge\right.$ $m)\left(1_{W M} \wedge T \wedge 1_{K}\right): W M K \wedge W M K \stackrel{1_{W M} \wedge T \wedge 1_{K}}{\longrightarrow} W M \wedge W M \wedge K \wedge K \stackrel{m_{W M} \wedge m}{\longrightarrow} W M K$ on $W M K$. Since the smash product of commutative ring spectra is a commutative ring spectrum, we have the following

Corollary 3.3. WMK is a commutative ring spectrum with multiplication $m_{W M K}$.

Consider the spectrum $W K=W \wedge K$ and a multiplication $m_{W K}$ on $W K$ defined by $m_{W K}=\left(m_{W} \wedge m\right)\left(1_{W} \wedge T \wedge 1_{K}\right)$ for the switching map $T: K \wedge W \rightarrow W \wedge K$. We have the split cofiber sequence

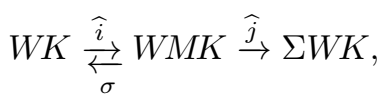

in which $\widehat{i}=1_{W} \wedge i \wedge 1_{K}: W K \rightarrow W M K$ and $\sigma$ denotes a splitting.

Lemma 3.5. $\widehat{i}_{W K}=m_{W M K}(\widehat{i} \wedge \widehat{i})$.

Proof. This follows from computation:

$$
\begin{aligned}
\widehat{i} m_{W K} & =\left(i^{\prime} \wedge 1_{K}\right)\left(m_{W} \wedge m\right)\left(1_{W} \wedge T \wedge 1_{K}\right) \\
& =\left(m_{W M} \wedge m\right)\left(i^{\prime} \wedge i^{\prime} \wedge 1_{K \wedge K}\right)\left(1_{W} \wedge T \wedge 1_{K}\right) \quad \text { by (3.1) } \\
& =\left(m_{W M} \wedge m\right)\left(1_{W M} \wedge T \wedge 1_{K}\right)(\widehat{i} \wedge \widehat{i})=m_{W M K}(\widehat{i} \wedge \widehat{i}) .
\end{aligned}
$$

Lemma 3.6. The spectrum $W K$ is a commutative ring spectrum with multiplication $m_{W K}$.

Proof. Apply $\sigma$ in (3.4) to Lemma 3.5, and we have

$$
m_{W K}=\sigma m_{W M K}(\widehat{i} \wedge \widehat{i}) .
$$

Then, noticing that $m_{W M K} T^{\prime}=m_{W M K}$ by Corollary 3.3 ,

$$
m_{W K} T \sigma m_{W M K}(\widehat{i} \wedge \widehat{i}) T=\sigma m_{W M K} T^{\prime}(\widehat{i} \wedge \widehat{i})=\sigma m_{W M K}(\widehat{i} \wedge \widehat{i})=m_{W K} .
$$

Here, $T: W K \wedge W K \rightarrow W K \wedge W K$ and $T^{\prime}: W M K \wedge W M K \rightarrow W M K \wedge W M K$ are the switching maps. The associativity of it is verified as follows:

$$
\begin{aligned}
m_{W K}\left(m_{W K} \wedge 1_{W K}\right) & =\sigma m_{W M K}(\hat{i} \wedge \hat{i})\left(m_{W K} \wedge 1_{W K}\right) \quad \text { by (3.7) } \\
& =\sigma m_{W M K}\left(m_{W M K} \wedge 1_{W M K}\right)(\hat{i} \wedge \hat{i} \wedge \widehat{i}) \quad \text { (by Lemma 3.5) } \\
& =\sigma m_{W M K}\left(1_{W M K} \wedge m_{W M K}\right)(\hat{i} \wedge \widehat{i} \wedge \widehat{i}) \quad \text { (by Corollary 3.3) } \\
& =\sigma m_{W M K}(\hat{i} \wedge \widehat{i})\left(1_{W K} \wedge m_{W K}\right) \quad \text { (by Lemma 3.5) } \\
& =m_{W K}\left(1_{W K} \wedge m_{W K}\right) .
\end{aligned}
$$

Consider the homomorphism

$$
\varphi_{W}:[K, K]_{*} \rightarrow[W K, W K]_{*}
$$

given by $\varphi_{W}(f)=1_{W} \wedge f$. Then, an easy computation shows

Lemma 3.8. The homomorphism $\varphi_{W}$ induces those of $\varphi_{W}: \operatorname{Mod}(K) \rightarrow \operatorname{Mod}(W K)$ and $\varphi_{W}: \operatorname{Der}(K) \rightarrow \operatorname{Der}(W K)$. 


\section{Construction of homotopy elements}

By definition, we see that $B P_{*}(W \wedge X)=B P_{*}(X) \oplus g B P_{*}(X)$ for a spectrum $X$ such that $B P_{s}(X)=0$ unless $q \mid s$, in which the degree of the generator $g$ is $p q-1$. This also implies the relation $E_{2}^{*}(W \wedge X)=E_{2}^{*}(X) \oplus g E_{2}^{*}(X)$ of the Adams-Novikov $E_{2}$-term. We apply this for $X=K_{u}$ and $\bar{K}_{u}$.

We begin with a general result corresponding to Oka's theorems [6, Th. 7.1, Th. 7.2] and [7, Construction III].

Proposition 4.1. Let $f \in \pi_{*}\left(W K_{u}\right)$ be an element such that $\eta_{*}(f) \equiv v_{2}^{s} \bmod \left(v_{1}\right)$ for the unit map $\eta$ of BP. Then,

1) for $n \geq 0$, there is an element $f_{n} \in \pi_{*}\left(W K_{2^{n} u}\right)$ such that $\eta_{*}\left(f_{n}\right) \equiv v_{2}^{s p^{n}}$ $\bmod \left(v_{1}\right)$, and

2) for $n \geq 1$ and a positive integer $u^{\prime}$ such that $u^{\prime} p \leq 2^{n-1} u$, there is an element $\bar{f}_{n} \in \pi_{*}\left(W \bar{K}_{u^{\prime}}\right)$ such that $\eta_{*}\left(\bar{f}_{n}\right) \equiv v_{2}^{s p^{n}} \bmod \left(p, v_{1}\right)$. Here, $W \bar{K}_{u}=$ $W \wedge \bar{K}_{u}$.

Proof. Put $f_{0}=f$, and suppose the existence of $f_{n} \in \pi_{*}\left(W K_{2^{n} u}\right)$. Then, $\kappa\left(f_{n}\right) \in$ $\operatorname{Mod}\left(W K_{2^{n} u}\right)$ for $\kappa$ in Theorem 2.1. By Theorem 2.2 and Lemma 3.8, $\delta^{\prime}=$ $\varphi_{W}\left(\delta_{2^{n} u}^{\prime}\right) \in \operatorname{Der}\left(W K_{2^{n} u}\right)$, and so we have $\kappa\left(f_{n}\right)^{p} \delta^{\prime}=\delta^{\prime} \kappa\left(f_{n}\right)^{p}$ by Theorem 2.3 . Thus we obtain a map $\widetilde{f}_{n+1}$, which makes the diagram

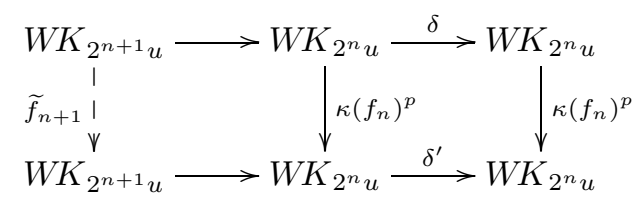

commute. Here, the rows of the diagram are the cofiber sequence of (2.4). Now put $f_{n+1}=\left(i_{W} \wedge i_{2^{n} u} i\right)^{*}\left(\widetilde{f}_{n+1}\right)$ to complete the induction.

To prove the second part of Proposition 4.1. By 1), we have $f_{n-1} \in \pi_{*}\left(W K_{2^{n-1} u}\right)$ for $n \geq 1$, and so an element $f_{n-1}^{\prime} \in \pi_{*}\left(W K_{u^{\prime} p}\right)$ for $u^{\prime} p \leq 2^{n-1} u$ (cf. [7, Construction I]). Theorem 2.7, Lemma 3.8 and Theorem 2.3 imply that

$$
\kappa\left(f_{n-1}^{\prime}\right)^{p} \delta^{\prime \prime}=\delta^{\prime \prime} \kappa\left(f_{n-1}^{\prime}\right)^{p}
$$

for $\delta^{\prime \prime}=\varphi_{W}\left(\delta_{u^{\prime}}\right)$, where $\delta_{u^{\prime}}$ is the map in (2.6). We then have a map $\widetilde{\bar{f}}_{n}$ fitting into the commutative diagram

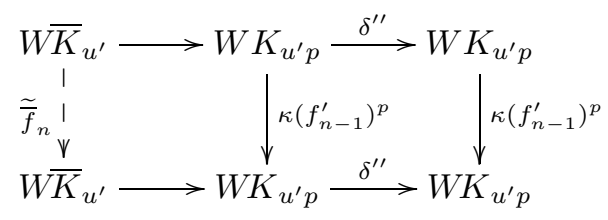

in which the rows of the diagram are the cofiber sequence in (2.6). Now the map $\bar{f}_{n}$ is obtained by $\left(i_{W} \wedge \bar{i}_{u^{\prime}} i\right)^{*}\left(\widetilde{\bar{f}}_{n}\right)$.

In [10], we show the following lemma:

Lemma 4.2 ([10, Lemma 2.11]). For $u>2$, there exists an element $\omega_{u} \in$ $\pi_{(p+2) q-1}\left(W K_{u}\right)$ such that $\left(j_{W}\right)_{*}\left(\omega_{u}\right)=i_{u} \alpha^{2} i \in \pi_{2 q}\left(K_{u}\right)$. Moreover, $v_{1}^{2} g \in E_{2}^{0}\left(W K_{u}\right)$ $=E_{2}^{0}\left(K_{u}\right) \oplus g E_{2}^{0}\left(K_{u}\right)$ detects it. 
A similar lemma follows from Lemma 2.9.

Lemma 4.3. For $u>1$, there exists an element $\bar{\omega}_{u} \in \pi_{2 p q-1}\left(W \bar{K}_{u}\right)$ such that $\left(j_{W}\right)_{*}\left(\bar{\omega}_{u}\right)=\bar{i}_{u} A \bar{i} \in \pi_{p q}\left(\bar{K}_{u}\right)$. Moreover, $v_{1}^{p} g \in E_{2}^{0}\left(W \bar{K}_{u}\right)=E_{2}^{0}\left(\bar{K}_{u}\right) \oplus g E_{2}^{0}\left(\bar{K}_{u}\right)$ detects it.

Proof of Theorem 1.9. By Proposition 4.1, we have elements $\left(f_{p^{i}, u}\right)_{n} \in \pi_{*}\left(W K_{2^{n} u}\right)$

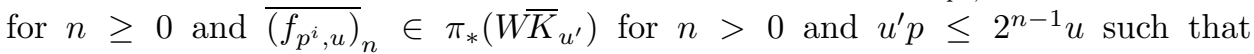
$\eta_{*}\left(\left(f_{p^{i}, u}\right)_{n}\right) \equiv v_{2}^{p^{i+n}} \bmod \left(v_{1}\right)$ and $\eta_{*}\left(\overline{\left(f_{p^{i}, u}\right)_{n}}\right) \equiv v_{2}^{p^{i+n}} \bmod \left(p, v_{1}\right)$. Consider the composites

$$
\begin{aligned}
B_{s p^{i+n} / 2^{n} u-r} & =\widetilde{\alpha}^{r-2}\left(j_{W} \wedge 1_{K}\right) \kappa\left(\left(f_{p^{i}, u}\right)_{n}\right)^{s} \omega_{2^{n} u} \quad(r \geq 2) \quad \text { and } \\
B_{s p^{i+n} / u^{\prime} p-r p, 2} & =\widetilde{A}^{r-1}\left(j_{W} \wedge 1_{\bar{K}}\right) \kappa\left(\left(f_{p^{i}, u}\right)_{n}\right)^{s} \bar{\omega}_{u^{\prime}} \quad(r \geq 1),
\end{aligned}
$$

where $\widetilde{\alpha}, \widetilde{A}, \omega_{u}$ and $\bar{\omega}_{u}$ are the elements of Lemmas 2.8 , 4.2 and 4.3. and $\kappa$ is the homomorphism in Theorem 2.1. Then, $\eta_{*}\left(B_{s p^{i+n} / 2^{n} u-r}\right) \in b\left(s p^{i+n} ; 2^{n} u-r, 1\right)$ and $\eta_{*}\left(B_{s p^{i+n} / u^{\prime} p-r p, 2}\right) \in b\left(s p^{i+n} ; u^{\prime} p-r p, 2\right)$. Since $j, j_{2^{n}}, \bar{j}$ and $\bar{j}_{u^{\prime}}$ correspond to $\partial_{1}, \partial_{1,2^{n} u}, \partial_{2}$ and $\partial_{2, u^{\prime} p}$, respectively, the elements $j j_{2^{n} u} B_{s p^{i+n} / 2^{n} u-r}$ and $\overline{j j}_{u^{\prime}} B_{s p^{i+n} / u^{\prime} p-r p, 2}$ are detected by elements of $\widehat{\beta}_{s p^{i+n} / 2^{n} u-r}$ and $\widehat{\beta}_{s p^{i+n} / u^{\prime} p-r p, 2}$, as desired.

\section{REFERENCES}

[1] Haynes R. Miller, Douglas C. Ravenel, and W. Stephen Wilson, Periodic phenomena in the Adams-Novikov spectral sequence, Ann. of Math. (2) 106 (1977), no. 3, 469-516. MR0458423 (56 \#16626)

[2] Shichirô Oka, A new family in the stable homotopy groups of spheres, Hiroshima Math. J. 5 (1975), 87-114. MR0380791 (52 \#1688)

[3] Shichirô Oka, A new family in the stable homotopy groups of spheres. II, Hiroshima Math. J. 6 (1976), no. 2, 331-342. MR0418096 (54 \#6140)

[4] Shichirô Oka, Realizing some cyclic $\mathrm{BP}_{*}$-modules and applications to stable homotopy of spheres, Hiroshima Math. J. 7 (1977), no. 2, 427-447. MR0474290 (57 \#13937)

[5] Shichirô Oka, Ring spectra with few cells, Japan. J. Math. (N.S.) 5 (1979), no. 1, 81-100. MR614695 (82i:55009)

[6] Shichirô Oka, Small ring spectra and p-rank of the stable homotopy of spheres, Proceedings of the Northwestern Homotopy Theory Conference (Evanston, Ill., 1982), Contemp. Math., vol. 19, Amer. Math. Soc., Providence, R.I., 1983, pp. 267-308. MR711058 (84j:55006)

[7] Shichirô Oka, Multiplicative structure of finite ring spectra and stable homotopy of spheres, Algebraic topology, Aarhus 1982 (Aarhus, 1982), Lecture Notes in Math., vol. 1051, Springer, Berlin, 1984, pp. 418-441, DOI 10.1007/BFb0075582. MR.764594 (86c:55017)

[8] Shichirô Oka, Derivations in ring spectra and higher torsions in Coker J, Mem. Fac. Sci. Kyushu Univ. Ser. A 38 (1984), no. 1, 23-46, DOI 10.2206/kyushumfs.38.23. MR736944 (85h:55019)

[9] D. Ravenel, Complex cobordism and stable homotopy groups of spheres, Second edition, AMS Chelsea Publishing, Providence, RI, 2004. MR0860042

[10] Katsumi Shimomura, Note on beta elements in homotopy, and an application to the prime three case, Proc. Amer. Math. Soc. 138 (2010), no. 4, 1495-1499, DOI 10.1090/S0002-993909-10190-9. MR2578544(2011c:55028)

[11] Katsumi Shimomura, The beta elements $\beta_{t p^{2} / r}$ in the homotopy of spheres, Algebr. Geom. Topol. 10 (2010), 2079-2090, DOI 10.2140/agt.2010.10.2079. MR2745666 (2011k:55009)

[12] Larry Smith, On realizing complex bordism modules. Applications to the stable homotopy of spheres, Amer. J. Math. 92 (1970), 793-856. MR0275429 (43 \#1186a)

Department of Mathematics, Faculty of Science, Kochi University, Kochi, 780-8520, JAPAN

E-mail address: katsumi@kochi-u.ac.jp 